\title{
Letter to the Editor re: ICP monitoring in children with TBI
}

\author{
Nadia Roumeliotis $^{1}$ (1) $\cdot$ Guillaume Emeriaud $^{1}$
}

Received: 4 January 2016 / Accepted: 6 January 2016 / Published online: 13 January 2016

(C) Springer-Verlag Berlin Heidelberg 2016

\section{Dear Editor:}

We thank Dr. Steinbok for his interest in the article "ICP Monitoring in Children: why are we not adhering to guidelines?". The main concern raised by Dr. Steinbok is that the monitoring of ICP is a weak recommendation in the current TBI guidelines, giving clinicians the opportunity to adapt their clinical practice on a case-by-case basis. We therefore should not say that ICP monitoring SHOULD be done in all children with severe TBI.

We fully agree that no strong evidence supports ICP monitoring in pediatrics. Guidelines, however, are developed in order to optimize and standardize practices. Although the recommendation regarding ICP monitoring is weak, due to lacking good-quality supportive data, ICP monitoring of a salvageable persistently comatose child remains standard of practice when clinically justified. Absence of ICP monitoring in these patients is frequently considered by experts as a nonadherence to guidelines [Vavilala CCM 2014].

We are, however, in full agreement with Dr. Steinbok that it is highly possible that ICP monitoring may not be beneficial in all children with severe TBI. This is precisely what our article attempts to illustrate. There are specific clinical situations where ICP monitoring is not undertaken (especially in moribund or rapidly improving comatose children), which should not be considered as non-optimal management. Further evidence is clearly needed to support ICP monitoring in less clear clinical situations (namely patients with "limit" Glasgow coma score and CT scan).

Further understanding as to what drives clinical decision making, and evaluating current practice, appears essential to support stronger recommendations in future guidelines for pediatric traumatic brain injury.
Nadia Roumeliotis

nadia.roumeliotis@gmail.com

1 Centre Hospitalier Universitaire Sainte-Justine, Montreal, Québec, Canada 